\title{
Exclusión digital ¿Cómo afecta en el desarrollo profesional y social de los habitantes del barrio Nueva Jerusalén?
}

\section{Digital exclusion how does it affect the professional and social development of the inhabitants of the Nueva Jerusalem neighborhood?}

\author{
Bismarck Alberto Arana Mite \\ Universidad Politécnica Salesiana, Ecuador \\ Karem Cadena Calle \\ Universidad Politécnica Salesiana, Ecuador
}

Autor para correspondencia: barana@ups.edu.ec, kcadenac@est.ups.edu.ec

Fecha de recepción: 05 de Julio de 2017 - Fecha de aceptación: 10 de Agosto de 2017

\begin{abstract}
Resumen
Este artículo presenta el análisis de los resultados sobre cómo afecta en el desarrollo profesional y social de los habitantes del barrio Nueva Jerusalén, la exclusión digital. Se explica de manera breve, la historia sobre la evolución, uso y aplicación de las nuevas tecnologías, esto permitirá al lector situarse en la realidad digital del país, generando conciencia crítica sobre el estado actual de las herramientas comunicativas e informativas que pueden ayudar al avance y desarrollo de la población. Las condiciones sociales y culturales han sido el mayor problema que sectores en riesgo han encontrado debido a la pobreza, bajos índices de educación, oportunidades laborales limitadas, no permiten abolir la miseria y donde se impone respetar las riquezas de los grupos mayormente globalizados.
\end{abstract}

Palabras Clave: tecnología; educación; oferta laboral; economía

\begin{abstract}
This article presents the analysis of the results on how it affects in the professional and social development of the inhabitants of the New Jerusalem, the digital exclusion. A brief history on the development, use and application of new technologies, this will allow the reader to be placed in the digital reality of the country, generating critical awareness about the current state of the communication tools and information that can help the advancement and development of the population. The social and cultural conditions have been the biggest problem areas at risk have been found due to poverty, low levels of education, limited job opportunities, do not allow the abolition of misery and where respect for the riches of the groups mostly globalized.
\end{abstract}

Key words: technology; education; labor supply; economy 


\section{Introducción}

Bajo las diferentes hipótesis surge la siguiente interrogante: ¿Cuáles son los factores que inciden en la exclusión digital de los habitantes del barrio Nueva Jerusalén de la ciudad Guayaquil?

La incidencia de las tecnologías y su aplicación en las instituciones educativas aceleran en gran medida la construcción de información y contenidos que se desarrollan a través de los nuevos canales y vías de fácil acceso. La presencia de las tecnologías digitales en el siglo XXI forma parte de la evolución cotidiana del ser humano en cuanto al desarrollo social, laboral, académico y cultural. (PNUD, 2016)

La idea que se tiene del uso y manejo de las nuevas herramientas tecnológicas radica en la aplicación correcta de estas, generando cambios en el conocimiento científico, de tal manera que se logre integrar en una sociedad de la información, la equidad social.

El desarrollo de una sociedad globalizada permite que todos quienes la integran formen parte del cambio, de tal manera que permita también el dominio eficaz de cada uno de los artefactos y servicios digitales presentados en un entorno mayormente industrializado, y con mejores ofertas productivas en el sector laboral y educativo.

De acuerdo con el Plan Nacional del buen vivir (2013-2017), presentado por el gobierno nacional, lo que el Estado busca en la actualidad es presentar desafíos que radican básicamente en la utilidad y usabilidad de las nuevas tecnologías en la sociedad. Con esto se busca garantizar los derechos del Buen Vivir, planteados en el capítulo segundo, sección primera de la Constitución política del Ecuador, vigente desde octubre del 2008.

Esto implica el empoderamiento digital de sectores globalizados, quienes con acceso libre y total a las Tecnologías de la Información y Comunicación alcanzan un estilo de vida superior, gozando de mejores plazas de trabajo, mayor calidad educativa y un estilo de vida digno en cuanto pueda otorgar la inclusión digital en comparación a los sectores vulnerables y con limitaciones económicas que impiden el crecimiento y desarrollo social paulatinamente con el avance tecnológico.

Según el Programa de las Naciones Unidas para el desarrollo (PNUD), la tecnología y las redes sociales facilitan nuevas formas y modalidades de organización y comunicación a nivel mundial.

\section{Brecha y exclusión digital}

El fenómeno brecha digital no es más que las desigualdades que existen entre las personas y sociedades por falta de acceso a las nuevas tecnologías, incluso, no basta con tener equipos y artefactos tecnológicos, sino saber, entender y conocer cómo utilizarlos para poder ser incluidos dentro del mundo virtual en la actualidad, de lo contrario surgiría el analfabetismo digital (Serrano \& Martínez, 2003). 
En muchos casos, las tecnologías digitales han impulsado el crecimiento, ampliando las oportunidades y mejorado la prestación de servicios. Sin embargo, su impacto agregado ha estado por debajo de las expectativas y sus beneficios se distribuyen de manera desigual (Grupo Banco Mundial, 2016).

DiMaggio et al. (2001) asumen que las desigualdades en el uso de Internet se producen entre los que tienen y los que no tienen acceso, y otros como (Bucy, 2000) y (Van Dijk, 2002) que definen las desigualdades no solamente como resultado del acceso potencial a Internet sino también como las diferencias en el uso de esta tecnología.

En este sentido, es importante destacar que la etapa modernista despliega sociedades diferentes: aquella que goza de riquezas empoderadas por el acceso a la información y conocimiento, dando paso a la inclusión digital de pocos; por otra parte, se encuentra la pobreza, aquel grupo imposibilitado al acceso de información e ilustración debido a su economía precaria generando exclusión digital, provocando riesgos que desencadenan en la inaccesibilidad de bienes y conocimientos. Desde esta perspectiva se origina la exclusión como un problema que va en aumento por factores económicos, educativos, sociales y culturales.

La brecha digital está relacionada con el acceso de las personas a las TIC en el que ignorar su utilización e implementación en la vida diaria, actualmente genera un alto índice de exclusión social. En el ámbito laboral se evidencia en gran medida la exclusión digital debido a que se requiere personas capacitadas y con habilidades para manipular un ordenador, acceder al internet con fluidez y, sobre todo, el conocimiento básico de las herramientas que facilite el acceso a la red.

Los sectores urbano-marginales del Ecuador que se ven limitados al acceso de las herramientas tecnológicas debido a factores económicos bajos aumentan el riesgo de perder las inversiones y oportunidades de empleo en comparación a los sectores que tienen una fuerza laboral más educada y mayormente globalizada.

Esto refiere a la diferencia tecnológica entre comunidades que tienen acceso a las TIC y aquellas que no. Por Tecnologías de Información entendemos el teléfono móvil, ordenadores, banda ancha, Internet y Software. Las diferencias pueden ser de tipo socioeconómico o sobre la capacidad para utilizar las Tecnologías de la Información de forma eficaz, debido a los distintos niveles de alfabetización y discapacidades (Sánchez Caballero, 2010).

Existen grandes problemas donde mayormente destaca el poderío económico de quienes logran costear los gastos básicos de educación y financiación económica, a diferencia de las personas que no gozan de bienes y se ven mermados de acceso tecnológico, de la posibilidad de integrarse a un sistema educativo y de las oportunidades laborales que se consiguen con una preparación académica indispensable para entrar en el mundo de los poderosos e ilustrados.

Según el Instituto Nacional de Estadísticas y Censos (INEC), el 31.4 \% de los 14.483.499 ecuatorianos usaron Internet en el último año. Se trata de una cifra que evidencia los avances que ha tenido el país en los últimos años en cuanto al acceso a esta tecnología, pero también revela 
que aún hay mucho por hacer para que la mayoría de la población ecuatoriana acceda a este beneficio.

Esto no significa que el $30 \%$ de la población tiene conexión fija a Internet en sus hogares, sino que muchos acceden a través de universidades, puestos de Internet, etc., ha señalado Fabián Jaramillo, Superintendente de Telecomunicaciones.

\section{Analfabetismo digital}

Según la definición de la Organización de las Naciones Unidas para la Educación, la Ciencia y la Cultura (UNESCO), es analfabeta funcional la persona que no puede emprender aquellas actividades en las cuales la alfabetización (saber leer y escribir) es necesaria para la actuación eficaz en su grupo y comunidad (Gutiérrez, 2003).

La implementación de las TIC en la actualidad se torna una función común para poder desempeñar los niveles de educación, conocimiento y aprendizaje en el mundo digital, con esto se da paso a la inclusión de todas las actividades en las cuales las vías electrónicas juegan un papel determinante en la formación y construcción de modelos digitales que permitan alcanzar la distribución de información eficaz para producir contenidos funcionales mediante el uso del internet.

Existen personas que no incorporan a su cotidianidad el uso de las herramientas tecnológicas porque no tienen bases que les permitan acceder a un mejor desempeño en el manejo de las mismas, sin embargo existen otros factores que interfieren para que los individuos no busquen interesarse en la utilización de los artefactos tecnológicos que les permita abrirse hacia nuevos conocimientos y de cierta manera, convertirse en personas autodidactas, es decir, aquellas que se educan por sí mismas y tratan de entender la funcionalidad de este sistema tecnológico.

Según el Informe de rendición de cuentas presentado por el Ministro de Telecomunicaciones y de la Sociedad de la Información (MINTEL), se redujo el analfabetismo digital del 21,4\% registrado en el 2012, al 14,4\%, en el 2014, En el 2015, el 12,2\% de las personas en el Ecuador son analfabetas digitales, 9,2 puntos menos que en el 2012 fomentando la equidad y la inclusión de la población, a través de capacitaciones en TIC, como aporte para el mejoramiento de la calidad de vida de los ciudadanos.

\section{Las TIC en Ecuador}

El término Tecnologías de Información y Comunicación, surge a través de los cambios presentados juntamente con el avance de los nuevos modelos tecnológicos generando un creciente impacto en cuanto al proceso de información se refiere, además dentro de este grupo se encuentran las actividades que incluyen el internet y los contenidos de comunicaciones que se desarrollan mediante el proceso de la información que se genera.

Cuando se habla de las TIC, se hace referencia a las técnicas utilizadas en base a mecanismos que faciliten el proceso de la comunicación e información digital volviéndose un 
elemento principal para las sociedades modernas. Sin embargo, un factor determinante para que se dé el desarrollo y evolución de las Tecnologías de la Información y Comunicación es el nivel socioeconómico, los niveles académicos y el alcance de las oportunidades laborales que son la base del desarrollo y sostenibilidad humana en la sociedad postindustrial en la que nos encontramos inmersos.

Según el Sistema de indicadores sociales del Ecuador (SIISE) el área rural mantiene la tasa de analfabetismo más alta (15.5\%) que la urbana (5.3\%). Según sexo, en el área urbana el porcentaje de mujeres analfabetas digitales (6\%) es mayor que el de los hombres $(4.5 \%)$, particular que se profundiza en el área rural: mujeres (18.1\%) y hombres (12.8\%).

La información proporcionada por el MINTEL, indica que el crecimiento y el acceso a las TIC se han dado gracias a la importancia de estas en el gobierno actual. Muestra de ello, es la intervención con laboratorios de computación en 8.937 entidades educativas fiscales del país, beneficiando a 2’110.447 estudiantes y 102.709 docentes, desde el 2007 hasta junio de 2014.

Sin embargo, actualmente existen sinnúmero de personas que no gozan de las grandes alternativas y posibilidades educativas que brindan estas herramientas informáticas en el ámbito de la comunicación y educación, solo acceden a esta posibilidad quienes están empoderados de riquezas, los grupos de estrato social alto, quienes pueden acceder a una educación sin límites, lo que se torna un desafío en cuanto a la inclusión y exclusión digital que trae consigo la evolución de las Tecnologías de Información y Comunicación generando así menos ascenso social e incremento de los índices de personas inmersas en la inequidad e inaccesibilidad de bienes tecnológicos por falta de economía, lo que genera riesgos y desequilibrio social con oportunidades limitadas al acceso educativo que repercuten al ascenso laboral.

\section{Metodología}

Para la recolección de información referente al objeto de estudio se empleó el enfoque mixto con un estudio representativo en función de las personas mayores de edad que habitan en el barrio Nueva Jerusalén. La muestra seleccionada fue no probabilística en la que destaca la selección por conveniencia o intencional, según (Kinnear \& Taylor, 1998) consiste en la selección de un elemento de la población que va a formar parte de la muestra basado en el criterio del investigador o entrevistador de campo.

Se utilizaron como herramientas la encuesta y la entrevista, y el programa SPSS para el procesamiento de datos estadísticos de toda la información recolectada. Se obtuvo un total de 71 encuestados que comprenden el número de observaciones que necesitábamos. Dicha muestra contiene las características que detallaremos a continuación.

La edad de las personas que participaron en la encuesta va desde los 18 hasta los 41 años en adelante, en cuanto al sexo de la muestra, 38 de las personas encuestadas son hombres y 33 son mujeres.

\section{Resultados}


Para el desarrollo de la investigación se analizó estadísticamente pregunta a pregunta en función de los datos proporcionados por la muestra seleccionada.

Sexo

Los resultados de la encuesta se presentan en el gráfico 1, donde se puede evidenciar que la muestra seleccionada de 71 personas está compuesta de la siguiente manera:

Hombres: 38 Personas Equivalente Al 54\%

Mujeres: 33 Personas Equivalente $\mathrm{Al} 46 \%$

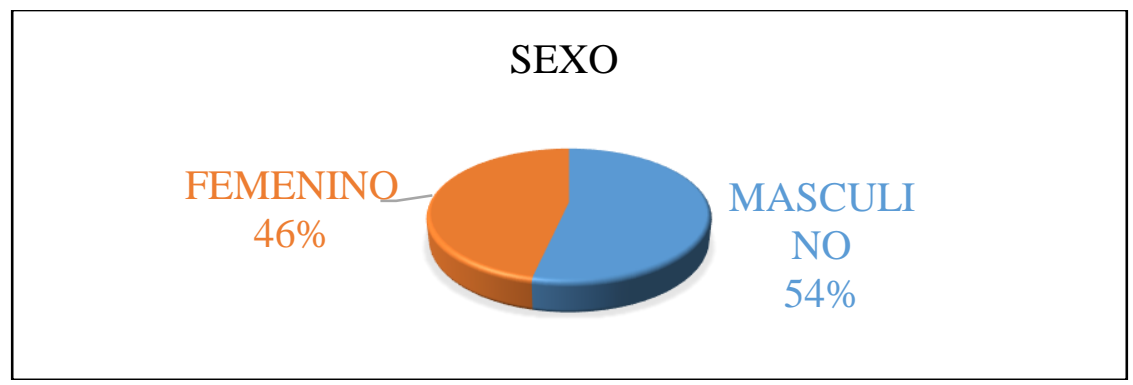

Gráfico 1.

Fuente: Elaboración propia

Edad

Con respecto a la edad de los encuestados se presentó 4 opciones determinadas en los siguientes rangos:

- De $18-25$ años

- $\quad$ De $26-30$ años

- $\quad$ De 31 - 40 años

- De 41 en adelante

Se obtuvo un mayor índice de personas dentro del rango de 41 años en adelante correspondiente al $52 \%$ de la población encuestada.

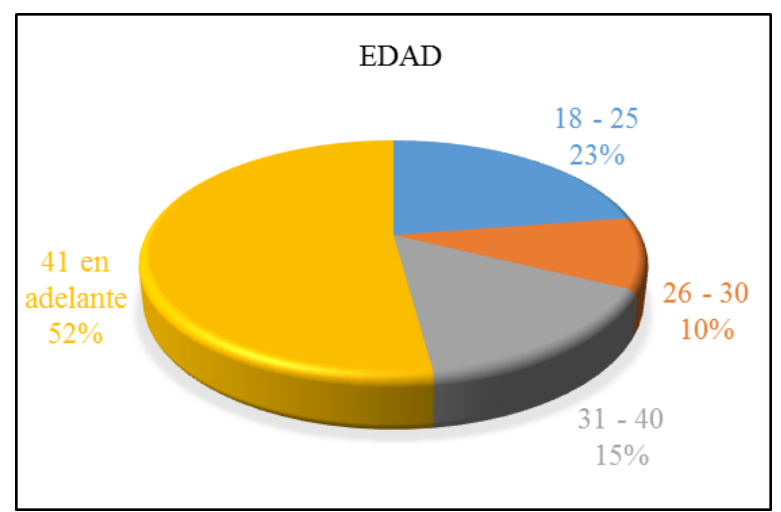

Gráfico 2.

Fuente: Elaboración propia 


\section{Nivel académico}

El nivel académico es un factor determinante para que la exclusión tome protagonismo y genere mayor índice de analfabetismo digital al no ser considerados parte de una sociedad actualmente digitalizada y con estructuras cognitivas puestas en práctica de manera eficiente.

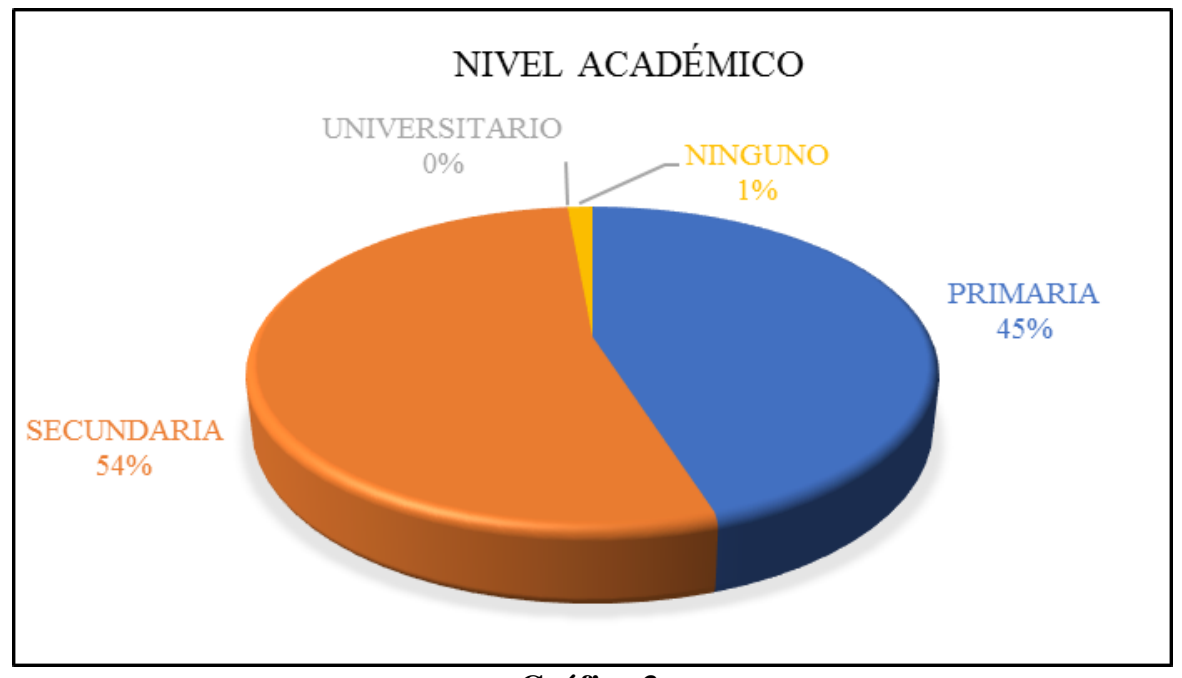

Gráfico 3.

Fuente: Elaboración propia

Estos valores determinan que el 100\% de la muestra carece de instrucción superior, dicho de otra manera, aquí encontraremos la mayoría de las falencias, lo que podría determinar las posibles causas y efectos de una exclusión digital y su repercusión en la sociedad a falta de una educación complementaria y tecnológica con una mejor preparación académica que permita insertar a las personas en un ambiente digitalizado e industrializado del siglo XXI.

En este caso es importante precisar que el nivel académico de los habitantes del barrio Nueva Jerusalén es medio - bajo lo que podría atribuirse a factores económicos limitados generando consecuencias de exclusión a falta de mejores niveles de preparación académicos.

\section{Accesibilidad tecnológica en instituciones educativas}

El acceso tecnológico en instituciones educativas complementa el aprendizaje en la actualidad, lo que no podríamos decir hace más de 25 años aproximadamente en aquellos centros educativos en los que existían la metodología tradicional de enseñanza.

Considerando que la mayor cantidad de personas encuestadas está en el rango de edad de 41 años en adelante equivalente al 52\% (Ver gráfico 2.) podemos concluir que el desconocimiento tecnológico se atribuye a la época y preparación académica que recibieron las personas, en este caso se evidencia el poco o nulo conocimiento tecnológico por parte de la población encuestada con un total del $68 \%$. 


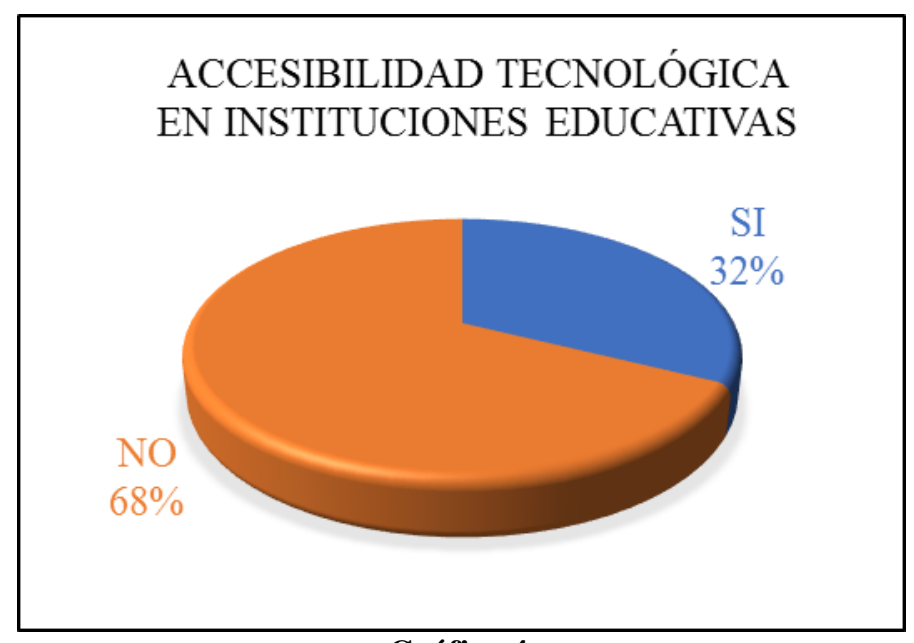

Gráfico 4.

Fuente: Elaboración propia

\section{Accesibilidad de equipos tecnológicos en viviendas}

Todas las personas encuestadas tienen al menos un equipo o artefacto tecnológico que les permite acceder a las nuevas herramientas y plataformas virtuales que ofrecen actualmente los medios digitales. Sin embargo, es válido recalcar que no todas las personas que conforman la muestra seleccionada tienen estos equipos conectados a internet, lo que hace que en muchas ocasiones pierda funcionalidad si se tratase de un artefacto básico y sencillo (llamadas, mensajería instantánea, etc.). En este estudio se desea analizar e interpretar es qué tipo de equipos tecnológicos tienen las personas del barrio Nueva Jerusalén y cuáles son utilizados con mayor frecuencia.

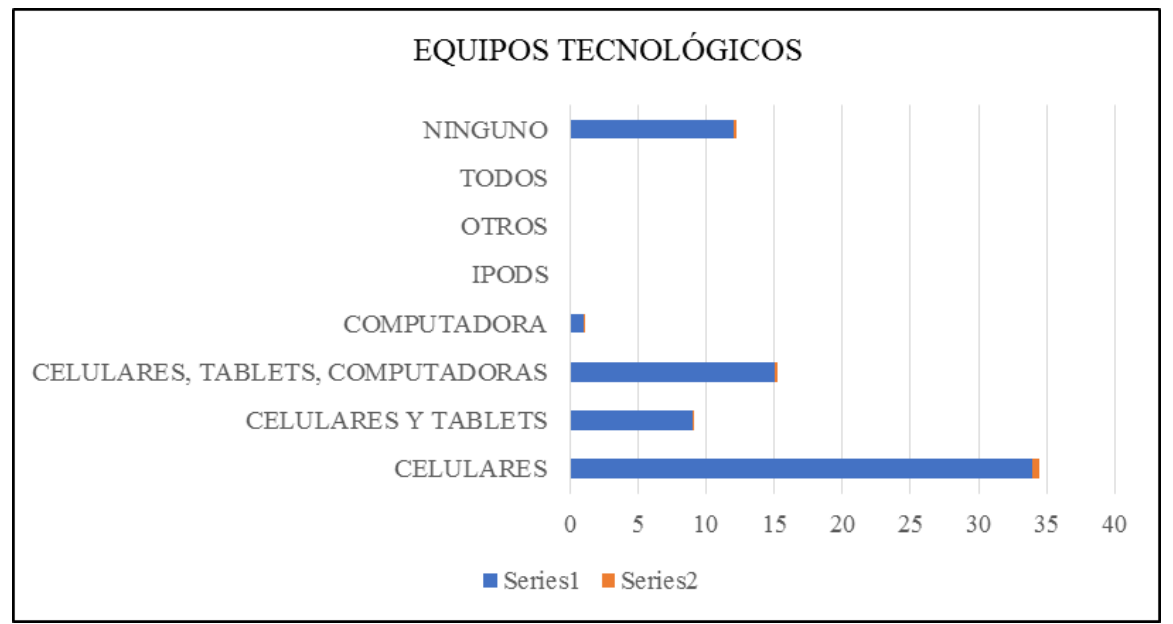

Gráfico 5.

Fuente: Elaboración propia

Se puede evidenciar que el equipo tecnológico con mayor incidencia en las personas encuestadas es el teléfono celular con un $48 \%$. Cabe precisar que hubo personas con más de un equipo tecnológico quienes marcaron hasta tres de las opciones dadas. Son: 
- Celulares, tablets, computadoras: $15 \%$

- Celulares, tablets: 9\%

\section{¿Sabe utilizar programas básicos de computación?}

Los programas básicos de computación están comprendidos de la siguiente manera: Word, Excel, Power Point.El 62\% de la población respondió que no sabe utilizar ninguno de los programas básicos de computación, mientras que el 38\% restante si sabe utilizar al menos uno de ellos

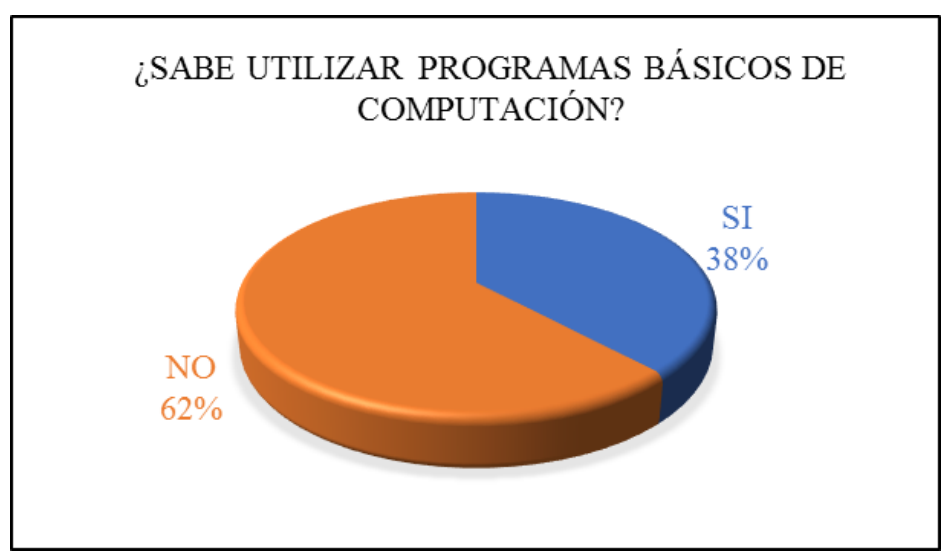

Gráfico 6.

Fuente: Elaboración propia

\section{Situación laboral}

El bajo nivel académico es uno de los factores que disminuye drásticamente las posibilidades de conseguir ofertas laborales en diferentes empresas, a medida que estas restricciones sociales aumentan, es más bajo el índice de desarrollo humano para los sectores vulnerables - urbano marginales como el barrio Nueva Jerusalén.

Existe una relación inversamente proporcional, a menor restricción presupuestaria, mayor preparación académica, por ende, aumentarán las posibilidades de inserción y empleo en la sociedad, en este caso y según los datos proporcionados por la población encuestada esto no se cumple debido a los índices bajos de preparación y economía del sector. Según el gráfico 7, el $49 \%$ de la población no trabaja, mientras que el $51 \%$ sí. 


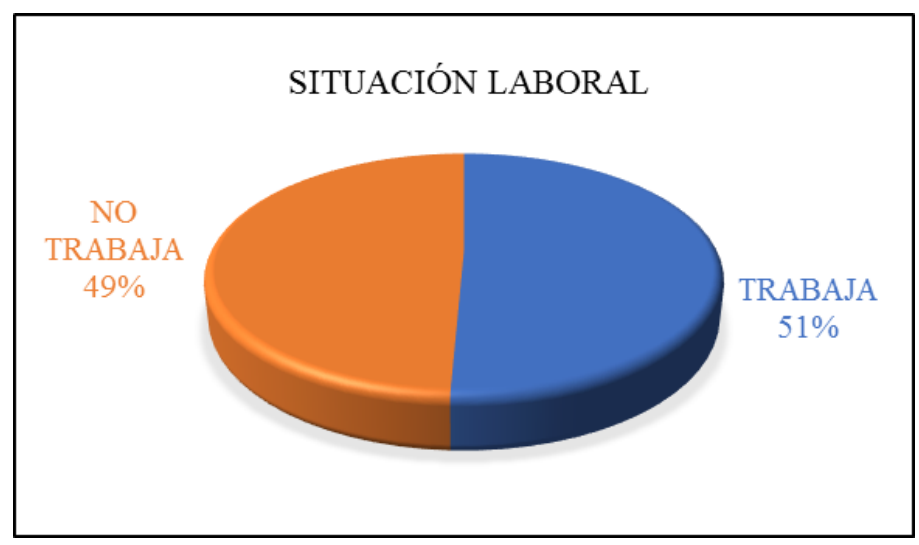

Gráfico 7.

Tipo de trabajo

Fuente: Elaboración propia

Tomando en consideración la pregunta número 7, se establecen 4 categorías en las que encasillamos el tipo de trabajo, son:

- Servicio doméstico, equivalente al $1 \%$ de la población

- Negocio propio, equivalente al 34\% de la población

- Empresa, equivalente al $15 \%$ de la población

- Ninguno, equivalente al $49 \%$ de la población que no trabaja

Estos valores demuestran que el subempleo es la mayor fuente de ingreso para hombres y mujeres del barrio Nueva Jerusalén.

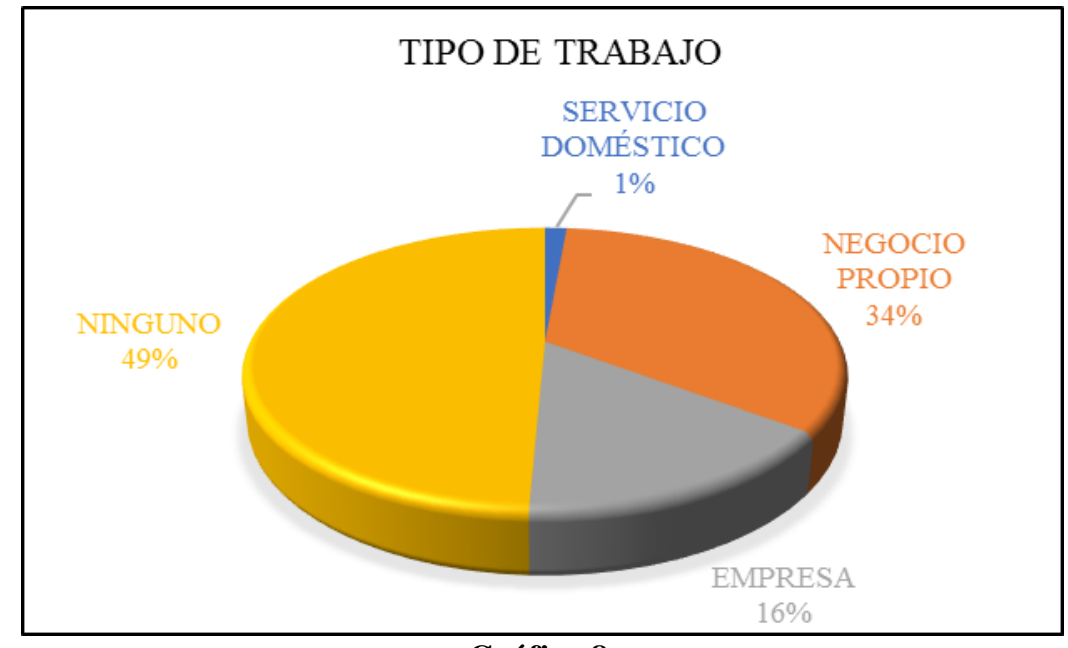

Gráfico 8.

Fuente: Elaboración propia

\section{¿Cree usted qué el desconocimiento tecnológico genera desempleo y falta de oportunidades?}

Del total de los encuestados, 63 personas equivalente al $89 \%$ coincidieron en que desconocer del uso y aplicación de las nuevas tecnologías en el siglo XXI ocasiona problemas a 
la hora de buscar empleos y generar mayores oportunidades en personas que se consideran analfabetas digitales al no saber cómo y de qué forma utilizar los equipos tecnológicos que mayormente se encuentran en las empresas/industrias. La mayor parte de los habitantes del barrio Nueva Jerusalén están sujetos al subempleo como medio de subsistencia, a pesar de que algunos han intentado a lo largo de sus vidas buscar empleos, han sentido el rechazo y exclusión debido a la falta de conocimientos tecnológicos y preparación académica, todo esto producto de una economía deficiente.

Por otra parte 8 personas equivalente al $11 \%$ consideran que desconocer de las nuevas tecnologías, uso y aplicación, no es impedimento para lograr campos de trabajo, algunos de los encuestados mencionaron que existen otras formas de promocionarse dependiendo de la actividad que desarrollen, muchas de estas tienen que ver con el subempleo como una forma de ayudarse (pesca, construcción, ebanistería) para lo cual no requieren tecnologías avanzadas para realizar sus trabajos sino simplemente ser conocidos en el sector donde viven.

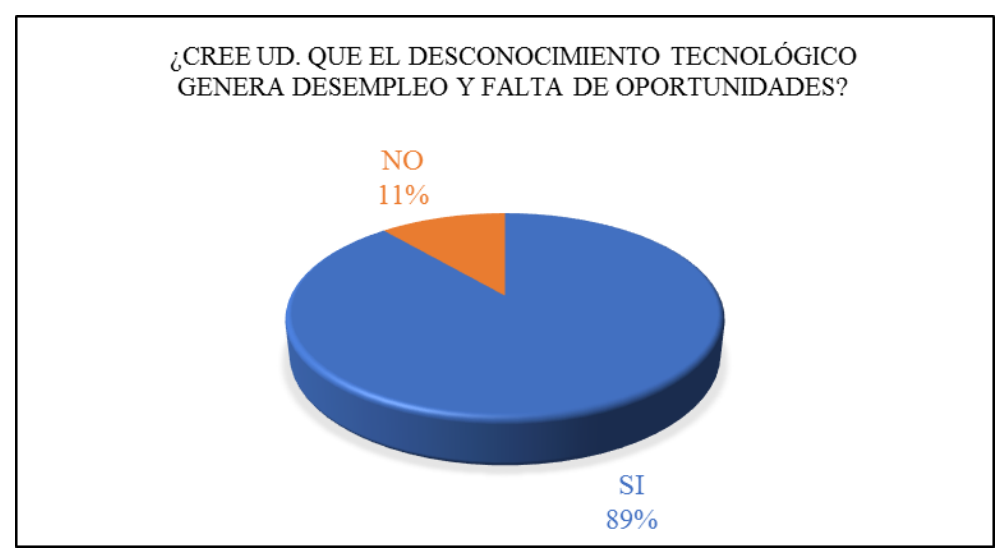

Gráfico 9.

Fuente: Elaboración propia

\section{Tiene hijos}

Para este análisis se tomó a 48 personas equivalente al $68 \%$ entre hombres y mujeres que tienen hijos descartando a las 23 que no representando el 32\%. Se toma en consideración que el número de hijos oscila desde $1,2,3 \ldots$ hasta 7 .

De estos valores se obtuvo un promedio de 2 hijos por familia encuestada, a continuación, mostraremos en la gráfica los porcentajes correspondientes. 


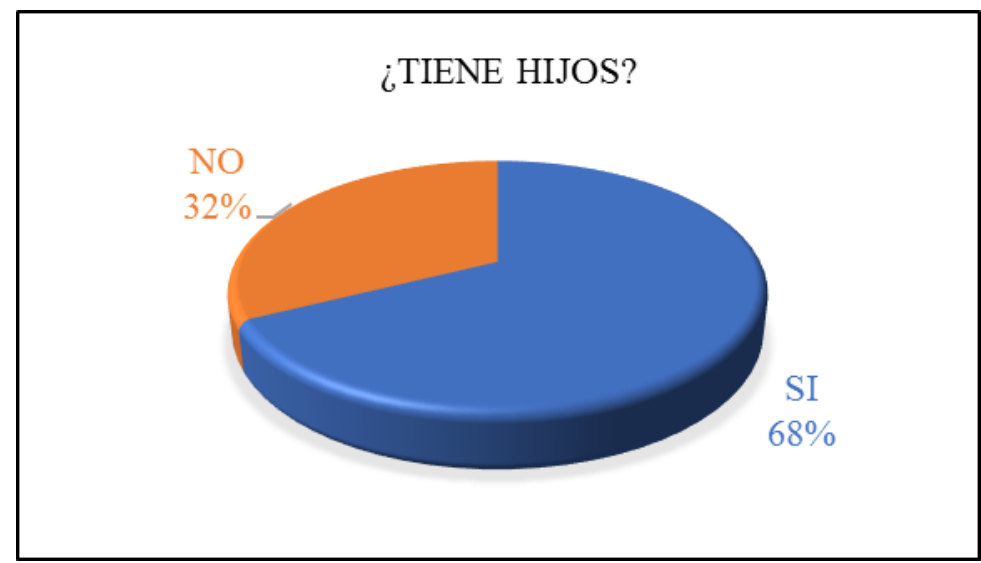

Gráfico 10.

Fuente: Elaboración propia

\section{¿En qué tipo de institución estudian sus hijos?}

Del total de encuestas (71) se obtuvo una muestra de 48 personas equivalente al $68 \%$, descartando a las 23 que no, esto equivale al $32 \%$, de la misma manera se procedió en la pregunta 11.

Como podemos evidenciar, la mayor parte de las personas tienen a sus hijos en instituciones públicas, lo que quiere decir que no gozan de una economía estable que les permita acceder a los beneficios que pueda otorgar una educación privada probablemente con mejores oportunidades académicas y tecnológicas, lo que permitirá a sus hijos mayor inserción social y con mejores índices de preparación.

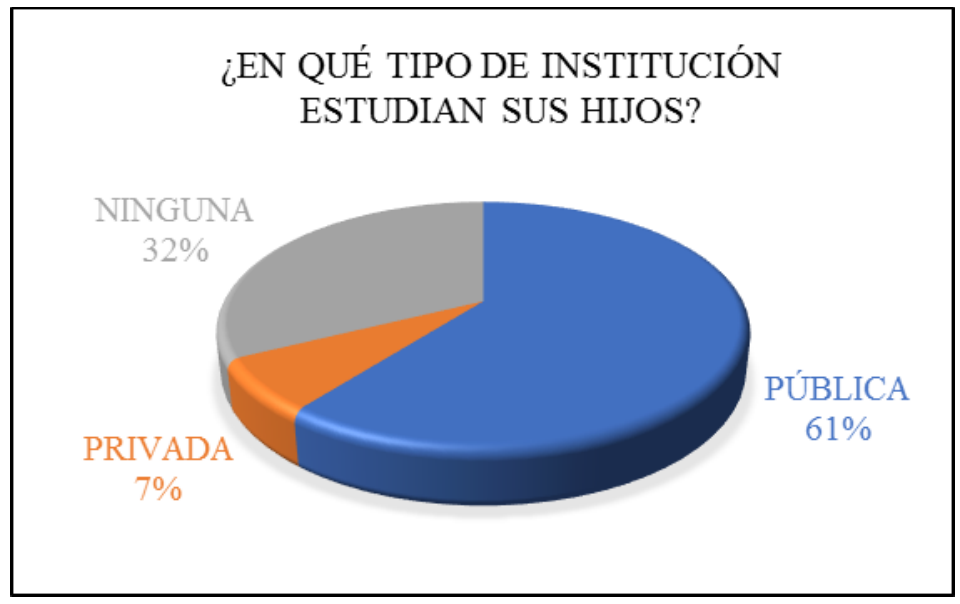

Gráfico 11.

Fuente: Elaboración propia

En las instituciones donde estudian sus hijos hay acceso a las nuevas tecnologías e internet

Si analizamos la pregunta 12 encontramos que los valores no guardan mucha diferencia, esto quiere decir que el $35 \%$ se trata de personas cuyos hijos si tienen acceso tecnológico en las 
instituciones educativas públicas y el $32 \%$ corresponde a las personas cuyos hijos no tienen acceso tecnológico.

Podemos evidenciar que a pesar de las políticas seccionales y de gobierno que buscan impulsar el avance, crecimiento y desarrollo de la población aún evidenciamos que no ha sido suficiente para alcanzar totalmente la alfabetización digital de los sectores urbano-marginales como el barrio Nueva Jerusalén a través de las instituciones públicas o privadas con un sistema educativo que les permita acceder en su totalidad a las nuevas herramientas y equipos tecnológicos, de ser así, podríamos hablar en un futuro de una sociedad digitalizada reduciendo los valores de exclusión por falta de preparación académica.

\section{¿Servicio de internet en domicilio es?}

Como observamos en la tabla de valores, el $50 \%$ de las personas que viven en el barrio Nueva Jerusalén se conectan a internet a través de la red Wi -Fi cercana a su sector y el otro 50\% paga una tarifa básica.

Estos valores son indicativos de que la población accede a internet sin importar los medios, lo cuestionable sería si saben utilizar esta herramienta como una puerta que les permita insertarse a la sociedad digitalizada del siglo XXI, o si son considerados al momento de conseguir empleo en empresas que soliciten el manejo de todas las plataformas y herramientas tecnológicas como requisito básico para su contratación. En este caso el Municipio de Guayaquil pretende masificar y expandir el uso de las nuevas herramientas tecnológicas y comunicativas, en las que se plantea como meta digitalizar a la población a través del alcance al internet con zonas Wifi libres en distintos sectores de la ciudad.

Estas alternativas presentadas no han sido suficientes para alcanzar que la población del barrio Nueva Jerusalén esté inserta en su totalidad en un ambiente tecnológico y digitalizado, existe un porcentaje significativo de personas que no acceden por factores económicos, educativos, sociales y culturales lo que afecta grandemente su desarrollo generando exclusión digital.

\section{Conclusiones y recomendaciones}

Concluido el análisis se determina que sí existe relación entre la exclusión digital y la poca inclusión laboral de los habitantes del barrio Nueva Jerusalén, a menor conocimientos y desempeño digital aumentará la tasa de desempleo en el sector, por ende, estaríamos comprobando un bajo índice de desarrollo humano y deficiencia académica que son los factores que influyen en el déficit económico del sector.

Mientras un individuo posea menos preparación académica, tendrá menos herramientas y posibilidades para enfrentar retos digitales siendo presa fácil de la exclusión. Las autoridades locales deben continuar y mejorar los proyectos en pro de una población mayormente digitalizada incentivando a los habitantes de los sectores más vulnerables a formar parte del cambio e inclusión. 


\section{Bibliografía}

Alva De La Selva, A. R. (2015). Los Nuevos Rostros De La Desigualdad En El Siglo Xxi: La Brecha Digital. Revista Mexicana De Ciencias Políticas Y Sociales, 60(223), 265-285. Https://Doi.Org/10.1016/S0185-1918(15)72138-0

Berrío, C., \& Candidato, Z. (2012). Entre La Alfabetización Informacional Y La Brecha Digital: Reflexiones Para Una Reconceptualización De Los Fenómenos De Exclusión Digital. Rev. Interam. Bibliot. Medellín (Colombia), 35(1), 39-53.

Bucy (2000) \& Van Dijk (2002). Umbrales Para La Determinación De La Brecha Digital: Comparativa Entre Regiones Desarrolladas. Recuperado El 03 De Diciembre De 2016, Http://Www.Scielo.Br/Pdf/Tinf/V26n2/0103-3786-Tinf-26-02-00125.Pdf

Caldera-Serrano, J. (2014). Umbrales Para La Determinación De La Brecha Digital : Comparativa Entre Regiones Desarrolladas Threshold For The Determination Of The Digital Breach: Comparative Among Developed Regions, 26(2), 125-132.

Dimaggio, (2001). Umbrales Para La Determinación De La Brecha Digital: Comparativa Entre Regiones Desarrolladas. Recuperado El 03 De Diciembre De 2016, Http://Www.Scielo.Br/Pdf/Tinf/V26n2/0103-3786-Tinf-26-02-00125.Pdf

Ecuador, A. N. (20 De Octubre De 2008). Constitución Del Ecuador. Recuperado El 30 De Noviembre De 2016, De Asamblea Nacional De La República Del Ecuador: Http://Www.Asambleanacional.Gob.Ec/Sites/Default/Files/Documents/Old/Constitucion_De _Bolsillo.

Ecuador, G. N. (2013-2017). Plan Nacional Del Buen Vivir. Recuperado El 05 De Diciembre De 2016, De Plan Nacional Del Buen Vivir: Http://Documentos.Senplades.Gob.Ec/Plan\%20nacional\%20buen\%20vivir\%2020132017.Pdf

Fundamedios (2012). Contra La Brecha Digital En Ecuador. Recuperado De: Http://Www.Fundamedios.Org/Contra-La-Brecha-Digital-En-Ecuador/

Grupo Banco Mundial (2016). Dividendos Digitales, Panorama General. Http://Documents.Worldbank.Org/Curated/En/658821468186546535/Pdf/102724-WdrWdr2016overview-Spanish-Webresbox-394840b-Ouo-9.Pdf

Gutiérez Martín, A. 2003. Alfabetización Digital. Algo Más Que Ratones Y Teclas. Barcelona. Gedisa. Isbn: 84-7432-877-2

Inec. (2013). Tecnologías De La Información Y Comunicación Tic`S. Ecuador En Cifras. Http://Www.Ecuadorencifras.Gob.Ec/Documentos/Web Inec/Estadisticas_Sociales/Tic/Resultados_Principales_140515.Tic.Pdf 
Inec. (2015). Tecnologías De La Información Y Comunicación Tic`S. Ecuador En Cifras. Http://Www.Ecuadorencifras.Gob.Ec/Documentos/Web-

Inec/Estadisticas_Sociales/Tic/2015/Presentacion_Tic_2015.Pdf

Johann Pirela, M., \& José De Jesús, C. V. (2014). El Desarrollo De Competencias Informacionales En Estudiantes Universitarios. Experiencia Y Perspectivas En Dos Universidades Latinoamericanas. Investigación Bibliotecológica: Bibliometría, Archivonomía E Información, 28(64), 145-172. Https://Doi.Org/10.1016/S0187-358x(14)70913-X

Kinnear \& Taylor, (1998). Capítulo Iii Metodología Udlap. Http://Catarina.Udlap.Mx/U_Dl_A/Tales/Documentos/Lemg/Lemaitre_W_N/Capitulo3.Pdf

Martínez Coral, P. (2014). Intervenciones Públicas De Inclusión Digital: Una Aproximación A Las Dimensiones Sociales Y Políticas De La Conectividad. Papel Político, 19(1), 61-76. Https://Doi.Org/10.11144/Javeriana.Papo19-1.Ipid

Matthew, S., \& Gabriela, C. $\quad$ (2013). Available In: Http://Www.Redalyc.Org/Articulo.Oa?Id=48028289001.

Mintel (2014). Tecnologías De La Información Y Comunicaciones Para El Desarrollo. Pág 123

Mintel (2015). Ecuador Redujo El Analfabetismo Digital Gracias A Las Políticas Impulsadas Por El Gobierno. Http://Www.Elciudadano.Gob.Ec/Ecuador-Redujo-El-Analfabetismo-DigitalGracias-A-Las-Politicas-Impulsadas-Por-El-Gobierno/ Pdf

Pnud (2016). Informe Sobre El Humano. Http://Hdr.Undp.Org/Sites/Default/Files/Hdr2016_Sp_Overview_Web.Pdf

Salvador, C., \& Pernia, R. (2007). Exclusión Social Y Tecnología.

Sánchez Caballero, M. 2010 Software Libre Y Accesibilidad. [Sitio Web] En: No Solo Usabilidad, No 9, 2010. Issn 1886-8592. [Consulta: 31 Enero 2013].

Sebastián, B. L., Magdalena, L., \& Nicolás, W. L. (2014). La Inclusión Masiva De Tecnologías Digitales En El Ámbito Escolar: Un Estudio Comparativo De La Apropiación De Tic Por Estudiantes De Clases Populares Y Clases Medias En El Marco Del Programa Conectar Igualdad En El Gran La Plata. Propuesta Educativa, 2, 86-92. Retrieved From Http://Www.Scielo.Org.Ar/Scielo.Php?Script=Sci_Arttext\&Pid=S199577852014000200010\&Lang=Pt

Serrano Santoyo, A.; Martínez E. 2003. La Brecha Dig

Straubhaar, J. D., Cuevas-Cerveró, A., Re-, H., \& Alarcón, R. O. (2016). Por Maria R. Osuna Alarcón, 30, 203-207. Https://Doi.Org/10.1016/J.Ibbai.2016.02.011

Toudert, D. E. (N.D.). Brecha Digital Y Perfiles De Uso De Las Tic En México: Un Estudio Exploratorio Con Microdatos, Iii, 167-200. 